Citation: B.R. Sarmiento, $M^{\mathrm{a}}$ Á. Hernández-Prados, N. Carbonell Bernal, M.C.Aguaded Gómez (2021)Alfabetización del profesorado en gamificación mediada por las TIC. Estado del arte. Media Education 12(1): 53-65. doi: 10.36253/me-10262

Received: December, 2020

Accepted: April, 2021

Published: May, 2021

Copyright: @ 2021 B.R. Sarmiento, $M^{a}$ Á. Hernández-Prados, N. Carbonell Bernal, M.C. Aguaded Gómez. This is an open access, peer-reviewed article published by Firenze University Press (http://www.fupress.com/me) and distributed under the terms of the Creative Commons Attribution License, which permits unrestricted use, distribution, and reproduction in any medium, provided the original author and source are credited.

Data Availability Statement: All relevant data are within the paper and its Supporting Information files.

Competing Interests: The Author(s) declare(s) no conflict of interest.

\section{Alfabetización del profesorado en gamificación mediada por las TIC. Estado del arte}

\author{
Teachers' literacy on gamification for the ICT. State of the art
}

\author{
Beatriz R. Sarmiento ${ }^{1}$, Ma Ángeles Hernández-Prados ${ }^{2}$, Noelia Car- \\ bonell Bernal 3 , María Cinta Aguaded Gómez ${ }^{4}$ \\ ${ }^{1}$ Universidad Internacional de la Rioja \\ ${ }^{2}$ Universidad de Murcia \\ ${ }^{3}$ Universidad Internacional de la Rioja \\ ${ }^{4}$ Universidad de Nebrija \\ E-mail: beatriz.rodriguezsarmiento@unir.net; mangeles@um.es; noelia.carbonell@unir. \\ net; maguaded@nebrija.es
}

\begin{abstract}
Recognizing the need for teacher literacy, highly supported by educational research, this work aims to carry out a reflective exercise on the educational potential of gamification mediated by Information and Communication Technologies (ICT) and its inclusion in the educational curriculum, offering all those aspects that a teacher who wishes to start in the gamification of their subject should know. To do this, following the methodological parameters of the state of the art, the concepts related to the term, such as learning through games or gamification, among others, are reviewed, as well as the features and characteristics of this active methodology and the educational impact it has in students, considering the perspective of different authors and the results of various investigations. Finally, some ICT tools and psychopedagogical guidelines are exposed that facilitate the implementation of gamification in the classroom by teachers. Every literacy process implies reaching the teaching competence level that contributes to the experiential transfer of knowledge to the classroom. With this intention this article has been constructed, wishing that it was inspiring and that it promoted the teaching methodological change.
\end{abstract}

Keyword: gamification, ICT, learning, teacher training.

Resumen. Reconocida la necesidad de alfabetización docente, altamente avalada por la investigación educativa, en este trabajo se pretende realizar un ejercicio reflexivo sobre el potencial educativo de la gamificación mediada por las Tecnologías de la Información y Comunicación (TIC) y su inclusión en el currículo educativo, ofreciendo todos aquellos aspectos que debe conocer un docente que desee iniciarse en la gamificación de su asignatura. Para ello se revisan, siguiendo los parámetros metodológicos del estado del arte, los conceptos afines al término, como el aprendizaje a través del juego o la ludificación entre otros, así como los rasgos y características propias de esta metodología activa y el impacto educativo que tiene en el alumnado, considerando la perspectiva de distintos autores y los resultados de diversas investigaciones. Finalmente, se exponen algunas herramientas TIC y orientaciones psicopedagógicas que faciliten la 
implementación de la gamificación en el aula por parte de los docentes. Todo proceso de alfabetización implica alcanzar el nivel competencial docente que contribuya a que el conocimiento se transfiera experiencialmente al aula. Con esta pretensión se ha construido este artículo, deseando que sea inspirador y que promueva el cambio metodológico docente.

Palabras clave: alfabetización docente, aprendizaje, gamificación, TIC.

\section{INTRODUCCIÓN}

La diversidad de aspectos educativos que penetran en el aula con la llegada del educando, son tantos que la tarea de educar lejos de ser una función sencilla y trivial, se ha convertido en una labor cada vez más compleja que demanda nuevas competencias y una mayor cualificación docente. Educar hoy día no es solamente transmitir a los alumnos los contenidos curriculares marcados legislativamente para cada etapa educativa, implica también atender e integrar en las aulas los recursos digitales emergentes. Algunos de ellos, como los blogs, wikis, flashmobs, cazas del tesoro, infografías, etc. pueden ser utilizados de forma dinámica por los docentes (Molina et al., 2015), despertando el interés de los estudiantes.

Vivir en una sociedad tecnologizada en la que el cambio es una seña de identidad de las instituciones y de la propia ciudadanía, tampoco ha facilitado la praxis educativa, demandando un perfil docente renovado, adaptativo, en constante cambio, en definitiva, un docente 3.0 acorde a la sociedad presente, que no tardará en cambiar y marcar nuevas tendencias y requisitos. El desafío actual radica por un lado, en favorecer la integración del educando en la sociedad del siglo XXI, lo que implica ser capaz de "localizar, analizar, seleccionar y utilizar los datos para la consecución de objetivos personales y sociales a fin de contribuir al desarrollo de mejores sociedades" (Guillén-Rascón et al., 2016, p.2); y por otro, cómo conseguir motivar en los aprendizajes escolares a las nuevas generaciones nacidas en una ciudadanía hiperconectada, hipertextual e hipercomunicada. A modo de respuesta, la gamificación unida a la tecnología, donde esta última será el elemento vehicular para desarrollar y poner en práctica los sistemas gamificados, constituye una posibilidad de mejorar la motivación del alumnado, aunque no la única (Gil \& Prieto, 2019).

En este sentido, la formación profesionalizadora que posibilite el ajuste de las funciones a las demandas es esencial para el desempeño de cualquier puesto laboral, pero en el caso de los docentes se vuelve aún más indispensable. De ahí que la tradicional alfabetización convive con otras formas de alfabetización digital, mediática e informacional, lo que se conoce como alfabetización múltiple. De todas las formas de alfabetización, la digital es la que más estrechamente está relacionada con la gamificación, e implica la habilidad "para entender las funciones de los medios, evaluar cómo se desempeñan aquellas funciones y comprometerse racionalmente con los medios para la auto-expresión" (UNESCO, 2011, p.185). Se trata, según Levis (2006), de la "capacidad para difundir, comprender y utilizar información en formatos múltiples a partir de fuentes de diverso origen generada, tratada y recibida por medio de computadoras" (p.79).

La importancia de la alfabetización digital ha sido puesta de manifiesto por diferentes autores. Si en la modernidad la alfabetización lingüística era considerada el motor impulsor de la integración social de la ciudadanía, en la sociedad actual, la alfabetización digital desempeña este mismo rol (Aparici \& Osuna-Acedo, 2010). Acaso no es esencial la capacitación tecnológica en las nuevas generaciones para favorecer su inclusión, especialmente cuando la información y el conocimiento son la seña de identidad de la sociedad y el motor de la economía. También se considera relevante para reducir la brecha generacional que se produce en la ciudadanía, especialmente en los espacios educativos en los que las interacciones son de diversas generaciones: docente-alumno o padres-hijos. Al respecto, García-Ávila (2017) señala que la alfabetización no se asocia a la posesión del recurso tecnológico, ni es un aspecto exclusivamente económico, más bien es educativo, ya que se asocia a las limitaciones o capacidades del sujeto para poder hacer un uso con criterio crítico-reflexivo de la información, herramientas y posibilidades que las tecnologías brindan al sujeto.

Esta alfabetización lejos de ser meramente contemplativa, acumulativa de saberes, se entiende como un aprendizaje instrumental que posibilita la capacitación del sujeto para crear y construir en una sociedad cada vez más digitalizada. En este sentido, el docente asume necesariamente un papel activo, hasta tal punto, que solamente se demuestra estar alfabetizado digitalmente cuando se muestran en la tarea educativa, de forma experiencial, las competencias adquiridas. Este papel activo de la alfabetización adquiere mayor protagonismo en el caso que nos ocupa: la gamificación, pues implica una transformación sustanciosa de los contenidos y competencias que se trabajan en las disciplinas para otorgarle un componente altamente motivador, el carácter de satisfacción y diversión que brinda el juego. 
Atendiendo a lo expuesto, la intención de este artículo es realizar una revisión del estado del conocimiento acerca de la alfabetización del docente en la gamificación mediada por las TIC, "que abra diálogos y dilucide sendas de acción” (Elías \& González, 2019, p.75), que permita retomar diversas conceptualizaciones, revisar los enfoques metodológicos y conocer enfoques innovadores, entre otros aspectos, a pesar de que no existe un consenso metodológico que distinga cuales son las estrategias o bien las etapas ineludibles para elaborar un estado del arte (Reyes, 2019). Según Gómez-Vargas et al. (2015), se inscribe en el marco de la investigación cualitativa documental, y adquiere tres modalidades: recuperar para describir, comprender y recuperar para trascender reflexivamente. El presente estudio se centra en la segunda, pues no busca balances e inventarios del conocimiento acumulado, al contrario, revisa la literatura con una finalidad hermenéutica, reflexiva, crítica y comprensiva. Se escogen unidades de información de las fuentes documentales y son sometidos a un proceso de revisión, reseña y descripción (Londoño et al., 2014). Se requiere una consulta amplia de documentos que permita establecer comparaciones, compresión del problema tratado, y ofrezca más de una alternativa de estudio (Molina-Montoya, 2005), en nuestro caso diversas posibilidades de transferencia al aula. Esta modalidad se encuentra presente en muchos otros estudios del arte que nos preceden, en el ámbito educativo de temáticas afines a la tratada, y que no han sido realizados desde los parámetros de revisión sistemática de las fuentes (Elías \& González, 2019; Guzmán \& García-Garduño, 2016; Molina-Ríos et al., 2017).

Siguiendo las indicaciones metodológicas, el presente trabajo tomar en consideración en primer lugar, la necesidad de seguir reflexionando sobre el concepto de gamificación, ya que si en el lenguaje común se siguen utilizando como sinónimos términos que no lo son, es señal de que es un aspecto a tratar en la alfabetización digital de la ciudadanía. Se aborda la red nomológica compuesta por los siguientes términos relacionados entre sí: ludificación, gamificación y aprendizaje basado en el juego. En segundo lugar, se realiza un recorrido por las ventajas y dificultades que se han asociado a la gamificación en los estudios previos, con la finalidad de promover la reflexión crítica hacia la misma y motivar actuaciones educativas coherentes. Para ello, se hace necesario que los docentes conozcan los aspectos metodológicos, así como las diferentes herramientas digitales que pueden facilitar el tratamiento de los contenidos curriculares de forma gamificada para conseguir un aumento de la motivación e implicación de los alumnos en el proceso de enseñanza y aprendizaje. Finalmente, a modo de conclusión, se destacan los beneficios de la gamificación mediada por las TIC, pero sin olvidar que se requiere un cambio de perspectiva para el éxito de esta metodología.

\section{APROXIMACIÓN CONCEPTUAL SOBRE GAMIFICACIÓN}

La gamificación no es una cuestión de modas pasajeras, más bien ha venido para quedarse. De hecho, ya se ha hecho hueco en nuestro lenguaje, en nuestras vidas, en los centros educativos, del mismo modo que hicieron otras tecnologías y metodologías precedentes. Su atención e importancia como metodología docente resurge actualmente a partir de la necesidad, en las instituciones educativas, de promover la motivación e interés de los estudiantes por el aprendizaje que compense la falta de compromiso educativo, el riesgo de fracaso y abandono escolar. Pero esta noción no ha estado exenta de ciertas contradicciones y confusión terminológica. Hablar hoy de "gamificación educativa", supone, por ejemplo, pensar en la interrelación del concepto de ludificación y aprendizaje. En el observatorio de palabras que La Real Academia Española (2019) tienen en estudio para su incorporación se contempla el término ludificación, cuya raíz latina significa juego (ludus), frente a la popularización del concepto de gamificación, que aunque nace asociado al término "game" de videojuego, una vez adaptado al ámbito educativo presenta una serie de diferencias ya que se encuentra más vinculado a la práctica educativa y al currículum.

A pesar de la tendencia existente por parte de los docentes, de aceptar estos dos conceptos como sinónimos, la gamificación busca relacionar los elementos curriculares con un proceso educativo más acorde a los aspectos formativos que los sujetos desarrollan vitalmente (Sánchez, 2015), mientras que la ludificación se refiere a la introducción de dinámicas que fomenten el aspecto lúdico en las aulas, pero sin utilizar contenidos curriculares ni fomentar el aprendizaje, es considerada como un proceso de "diversión" dentro de las aulas.

La finalidad educativa en el juego y la gamificación constituye otro elemento de diferenciación, mientras el objetivo principal del primero es divertirse o adquirir y mejorar una habilidad que se está practicando como sucede en los juegos de simulación, en el segundo, los elementos lúdicos son una alternativa de realización de la tarea, no son parte de las habilidades a mejorar (Deterding et al., 2011). Su objetivo principal es motivar ciertas acciones, para dotar de mayor comprensión los aprendizajes curriculares. Según Deterding et al. (2011), la gamificación se diferencia del juego, principalmen- 
te por cuatro elementos característicos: el concepto de game no es equiparable al de play; no se gamifica a partir del juego en su totalidad, si no en partes (element); un buen diseño aumenta la motivación de los alumnos, y como consecuencia se incrementa la implicación, y por último, non-game-context, referido a que no se trabaja desde el juego, sino que están inmersos en el contexto educativo. Es importante diferenciar también los modos de gamificación que pueden implementar los docentes en el aula: la superficial o de contenido, caracterizada por ser una acción corta y puntual; y la estructural o profunda, presente en la programación completa de un curso (Garone \& Nesteriuk, 2019).

La gamificación implica la aplicación de recursos, dinámicas, mecánicas y/o elementos típicos del juego en contextos que en principio son ajenos al juego y se categorizan de no lúdicos, como puede ser el aula (Rivero, 2017; Teixes, 2014), o en sus diferentes aplicaciones. Puede ser considerada como una demostración práctica de que el proceso educativo puede combinarse con una parte lúdica y motivadora de tal forma que se consigue aumentar los niveles de participación e interacción entre los alumnos, así como la satisfacción hacia los procesos de enseñanza-aprendizaje, lo que acaba influyendo en sus resultados académicos y sociales.

En este sentido, captar el interés y despertar la motivación estudiantil es un reto docente que puede verse favorecido gracias a los juegos, porque el propio diseño del juego genera el engagement (Hamari et al., 2016). Los juegos cautivan y divierten, principalmente a los jóvenes quienes invierten gran parte de su tiempo libre jugando. Valiéndonos de ese interés, se deberían utilizar sus dinámicas en el proceso de enseñanza-aprendizaje con la intención de transformarla en algo más divertido, animado y atractivo para que seduzca y conquiste a los alumnos (Quintanal, 2016). Además, contribuye a que el alumno disfrute de un aprendizaje experiencial e inmersivo, basado en la motivación y el trabajo colaborativo, consiguiendo que el proceso de enseñanza aprendizaje resulte más efectivo para estos alumnos (Bonilla del Río et al., 2018).

Relacionado con el término gamificación, una de las metodologías que están tomando más importancia en las aulas es el aprendizaje basado en el juego (ABJ), actualmente esta forma de trabajo está incluida dentro de las denominadas pedagogías emergentes. Al respecto, Qian y Clark (2016), afirman que la utilización del ABJ en las aulas no solo promueve el aprendizaje, sino que además potencia el desarrollo del pensamiento crítico y la resolución de problemas que se incluyen en las denominadas "destrezas del siglo XXI". La diferencia fundamental entre las dos metodologías activas se basa en que en el
$\mathrm{ABJ}$ se utilizan como herramientas de apoyo juegos, ya existentes, con fines educativos en los que el resultado, además del aprendizaje, será el ganar o perder ese juego en la sesión en que se haya introducido, mientras en la gamificación se incluyen elementos de juegos en el contexto educativo a lo largo de todo el proceso de aprendizaje (Chaves-Yuste, 2019).

\section{EL POTENCIAL EDUCATIVO DE LA GAMIFICACIÓN}

En la actualidad, la implementación de las TIC a través de la gamificación en el ámbito educativo, se ha convertido en una tarea compleja, a la vez que necesaria, puesto que cuenta con una serie de ventajas, que los docentes deben conocer para poder aplicarla correctamente en las aulas, pero sin olvidar, que a su vez también cuenta con una serie de inconvenientes que deben minimizarse gracias al buen uso de la metodología. De ahí que los docentes se encuentren en la obligación de proponer e instaurar una correcta utilización de las nuevas tecnologías en las aulas con métodos educativos tales como el de la gamificación, que sirvan de modelo de un uso responsable y educativo de las TIC.

A la hora de aplicarla en las aulas se debe tener en cuenta que el principal objetivo es lograr una acción formativa provechosa superando barreras como la falta de concentración o motivación, nivel insuficiente de conocimientos y habilidades o el aprendizaje en solitario. Gracias a una adecuada sistematización en su aplicación se pueden superar dichas dificultades potenciando el aprendizaje colaborativo, facilitando las múltiples oportunidades que fomentan el aprendizaje por ensayo-error, visibilizando los avances obtenidos y adaptando los ritmos individuales, aspectos que desembocan en la disminución del sentimiento de fracaso y el aumento de la motivación y concentración del estudiante (Teixes, 2015).

La gamificación, por lo tanto, enriquece el proceso de enseñanza - aprendizaje y facilita la consecución de sus objetivos (Bunchball, 2010). Desde un punto de vista psicopedagógico, la literatura nos muestra diversos estudios acerca de las posibilidades que puede ofrecer. Por un lado, Contreras y Eguia (2016) afirman que el uso de esta metodología favorece el desarrollo de competencias tan importantes para los alumnos como las habilidades sociales, habilidades cognitivas, toma de decisiones o las funciones ejecutivas, entre otras. Otros autores como Llorens-Largo et al. (2016) sostienen que, además de estos beneficios en las habilidades de los alumnos, no se debe olvidar que su uso en las aulas al tener un fuerte componente lúdico fomenta la motivación e implicación 
de los alumnos. En esta línea, Apostol et al. (2013) aclaran que la gamificación tiene un efecto en la motivación tanto extrínseca como intrínseca. El campo de la neurodidáctica aporta evidencias de que genera los efectos mencionados al demostrar que esta metodología activa el sistema de recompensa cerebral implicado en el aprendizaje (Rackwitz, 2012; Miller, 2013).

Por todo ello, su uso puede ser considerado una alternativa factible para solucionar algunos de los problemas más comunes que presentan los alumnos en las aulas al poder adaptarse a los distintos niveles educativos y diversidad del alumnado, además de proporcionar una mejora del aprendizaje significativo gracias a la implicación emotiva del alumno al aumentar su motivación y disminuir la percepción de fracaso (Hammer \& Lee, 2011); una mejora de los procesos cognitivos de los alumnos mediante la organización de la dificultad de los contenidos por niveles, desarrollando así las funciones ejecutivas, la atención, resolución de conflictos, reflexión y toma de decisiones (Llorens-Largo et al., 2016); una mejora del componente social al requerir de un trabajo colaborativo y una participación activa (Fernández-Arias et al., 2020); así como el desarrollo de competencias fundamentales de aprendizaje tales como aprender a aprender, trabajo autónomo y competencias trasversales.

Si bien la gamificación se puede llevar a cabo al margen de las Tecnologías de la Información y la Comunicación, no se puede perder de vista que la labor educadora se debe adecuar a la sociedad en la que se encuentra inmersa el estudiante, y en la sociedad del siglo XXI las TIC "conforman una parte significativa del mundo transmedia de los jóvenes y forman parte de su cultura" (Prieto-Andreu, 2020, p.75), así se han venido a denominar sabios digitales (Prensky, 2009) dada la cotidianeidad con la que emplean la tecnología desde la infancia. Por tanto, será necesario para una correcta gamificación del aprendizaje crear materiales educativos acordes a las nuevas experiencias y formas de expresarse en la sociedad digital.

A partir de la revisión realizada por Prieto-Andreu (2020) se observa que son muchos los estudios que demuestran los efectos positivos de la gamificación basada en el uso de las TIC en el proceso de enseñanza-aprendizaje en distintos niveles de educación afectando desde la motivación de los estudiantes (Cózar \& De Moya, 2013) hasta el rendimiento académico (Mominó \& Singalés, 2016). En este sentido, la revisión sistemática llevada a cabo por Torres-Toukoumidis et al. (2016) remarca la mejora en el proceso de aprendizaje de la lectura a través del uso de videojuegos como herramienta pedagógica. Un estudio llevado a cabo por Naismith et al. (2004) puso de manifiesto los beneficios en el proceso de enseñanza-aprendizaje de utilizar dispositivos móviles al permitir la construcción del conocimiento partiendo de aprendizajes previos, el diseño de actividades en contextos más reales y significativos, el aprendizaje basado en la confluencia de educación formal e informal y el servir como herramienta para ampliar y proporcionar recursos y materiales al alumnado. Por su parte, los resultados de la experiencia en educación primaria desarrollada por Villena et al. (2018) sobre narrativa dinámi$\mathrm{ca}$ en grupos interactivos con soporte web, muestran una mejora tanto en el aprendizaje de contenidos y habilidades de búsqueda y selección de información esenciales en el trabajo grupal, como en el interés, la motivación y la participación de los estudiantes. Igualmente, Pertegal y Lorenzo (2019) corroboran la mejora de la motivación, la atención y el aumento del tiempo de asistencia a clase del alumnado universitario a través de la incorporación del juego on-line mediante Kahoot a la asignatura.

Por tanto, los estudios muestran que son muchas las ventajas de introducir la gamificación a partir de las TIC en el ámbito educativo, pero ello no debe desviar nuestra atención de las desventajas que la utilización de esta metodología lleva aparejadas con el objetivo de desarrollar sistemas gamificados que las eviten y sean efectivos para la construcción del conocimiento. Para ello los docentes deben partir de la programación y diseño de la experiencia gamificada. En primer lugar, hay que recordar que se usan elementos de juego en una situación de aprendizaje, por lo que el juego no debe distraer del objetivo educativo. Otro aspecto a tener en cuenta se relaciona con las características del alumnado al que va dirigido, así por ejemplo, podría desembocar en comportamientos altamente competitivos (Furdu et al, 2017), que podrían producir efectos adversos en estudiantes poco competitivos como ansiedad y falta de implicación en el proceso de aprendizaje. Del mismo modo, cuando los premios recibidos en el sistema gamificado promueven exclusivamente los niveles de estatus, nos podríamos encontrar con estudiantado que se desinterese dado que no siente la necesidad de obtener reconocimiento para avanzar en su aprendizaje, o al contrario si la respuesta dada se basa en una rutina hedonista, en el momento que no se reciba la recompensa se producirá la desmotivación (Gil \& Prieto, 2019), ya que como apuntan estudios como el de Dicheva et al. (2015) la motivación intrínseca no se logra en todo tipo de sistema gamificado. Un modo para solventar estos problemas pasa por recompensar los esfuerzos y no los resultados (Kiryakova et al., 2014), así como por involucrar a los destinatarios en todo el proceso y no utilizar la gamificación como práctica obligada o dinámicas autoritarias, si no que "deberían generarse las reglas de todo juego 
social: de forma democrática, entre todos y desde abajo hacia arriba" (Escribano, 2013, p. 67). Todo ello creará un clima de cooperación para lograr la meta propuesta. Es importante también tener en cuenta los efectos que pueda tener en la educación en valores, más allá de la competitividad y el placer hedónico ya comentados, los entornos donde se lleva a cabo la experiencia pueden reforzar determinados estereotipos si no se cuidan los roles, lenguaje e información que se presentan (Fernández-Arias et al., 2020).

Una vez plasmados los puntos fuertes y débiles de la utilización de la gamificación a través de las TIC en las aulas, se observa que el empleo de esta metodología se ha convertido en un instrumento útil para conseguir que el proceso de enseñanza-aprendizaje del alumnado se adapte a la realidad educativa y social en la que estamos inmersos, consiguiendo aumentar la motivación de estos alumnos mediante una metodología no tradicional pero efectiva.

\section{ASPECTOS METODOLÓGICOS DE LA GAMIFICACIÓN}

Partiendo de la identificación de las necesidades de aprendizaje que se derivan de la sociedad actual, y de la forma en la que aprenden las nuevas generaciones, los docentes deben diseñar los ambientes educativos que favorezcan procesos de enseñanza-aprendizaje. Para Llorens-Largo et al. (2016) la estrategia metodológica que propicia la motivación, implicación y diversión del estudiante, mientras consigue los objetivos propios del proceso de aprendizaje, requiere diseñar ambientes lúdicos en los que se sienta involucrado, pueda tomar decisiones, aprenda a asumir nuevos retos, y a ser reconocido por sus logros. Por tanto, no cabe duda de que uno de los rasgos más señalados de la gamificación es el hecho de que se trata de una metodología activa que pone el protagonismo en el educando. Por ello, los docentes deben promover actuaciones en las que el estudiante goce de libertad y autonomía sobre su propio aprendizaje. Al respecto, Díaz-Cruzado y Troyano (2013) afirman que la utilización de videojuegos a través de internet, como modalidad gamificada, emplea técnicas provenientes de la educación y la psicología que fomentan de forma positiva el aprendizaje autónomo del alumno. Se debe aclarar que la autonomía como aspecto más intrapersonal e individualizado no está reñida con el trabajo colaborativo que acompaña a la gamificación. Como podemos comprobar en el estudio de Lamoneda et al. (2020), el trabajo en grupo por parte de los alumnos mediante el uso de la gamificación es una de sus señas de identidad, puesto que esta metodología de aprendizaje no obtiene los mismos resultados si es el alumno quien lo realiza de forma autónoma si no que debe trabajar con sus compañeros.

Para diseñar estos ambientes gamificados, el docente debe tener en cuenta los siguientes elementos: medio físico (aula de clase, patio, parques), la dotación de recursos (el tiempo y medios necesarios), su distribución en un espacio determinado, así como otros componentes temporales referidos a la planificación, funcionales donde lo lúdico debe establecerse como pauta de conducta, y relacionales centrados en los ajustes de roles del maestro y estudiante y las conductas a desempeñar (Acevedo et al., 2019; Iglesias-Forneiro, 2008).

El docente debe partir de las características del alumnado que tomará parte en el sistema gamificado para que esta metodología obtenga un resultado exitoso. Para ello debe conocer qué motiva a sus estudiantes en el ambiente lúdico, o lo que es lo mismo qué tipo de jugadores son, ya que de ello dependerán la planificación, ambiente y elementos de juego. En palabras de Marczewski (2015), podemos identificar 6 tipos de jugadores: socializadores, motivados por la relación y vinculación social con los otros; espíritus libres, que buscan la autonomía y expresión personal por medio de la creación y la exploración; triunfadores, motivados por el dominio de competencias, les mueven los retos y superarse a ellos mismos con el objetivo de obtener cierto estatus; filántropos, pretenden ayudar desinteresadamente a los demás; jugadores tradicionales, cuya motivación es totalmente extrínseca guiada por los premios obtenidos; por último, están los disruptores, con un desempeño orientado al cambio del sistema tanto en positivo (modders) como en negativo (trolls). Evidentemente, no estamos hablando de estilos puros, sino de tendencias por lo que habrá rasgos muy marcados de un tipo y menos marcados del resto de jugadores en cada alumno.

Un modo de conocer el tipo de jugadores que conforma nuestro alumnado es mediante la aplicación de la Escala Gamification User Type Hexad Scale ${ }^{1}$ validada para población española (Tondello et al., 2019) o bien creando un sistema que tenga en cuenta las características de los jugadores más comunes (filántropos, socializadores, espíritus libres y triunfadores), es decir, los tipos basados en la motivación intrínseca (Tondello et al., 2016). Concretamente, debería primar el trabajo colaborativo orientado a un fin, la vinculación social, la autonomía, la creatividad y el desarrollo de competencias.

Pero ¿cómo se desarrollan esos sistemas gamificados, qué elementos se deben tener en cuenta? Antes de

\footnotetext{
${ }^{1}$ https://gamified.uk/UserTypeTest2016/user-type-test.php?q=1\&lan$\mathrm{g}=\mathrm{es \# .X9iNMS8rxN0}$
} 
sugerir qué elementos de juego se podrían asociar a cada tipo de jugador, para así motivar la conducta deseada y mejorar el proceso de enseñanza-aprendizaje, es necesario detenerse en la presentación y agrupación de dichos elementos en componentes, mecánicas, dinámicas y estética (Fernández-Arias et al., 2020; Rodríguez-García \& Santiago-Campión, 2015; Teixes, 2015). Las mecánicas se relacionan con el modo de jugar y permiten visibilizar el progreso en el juego, las más comunes son puntos, medallas, monedas, clasificaciones, reglas, retos o misiones. Las dinámicas surgen de la puesta en práctica de las mecánicas y su objetivo es involucrar al jugador en el sistema, basándose, como apuntan Werbach y Hunter (2012) en el reconocimiento, consecución de objetivos, autosatisfacción, entre otros deseos básicos de las personas. Se englobarían aquí recompensas, competición, socialización, aprendizaje, identidad, estatus, diversión o altruismo. Los componentes se refieren a las implementaciones concretas a la hora de poner en marcha las mecánicas y dinámicas, se incluyen es este tipo de elementos los logros, colecciones, combates, niveles o avatares. Por último, la estética se refiere al diseño del sistema gamificado e influye directamente en las emociones, sensaciones y experiencias que emergen en el jugador, según Hunicke et al. (2004) se clasificarían en narrativa, reto, sensación, fantasía, camaradería, descubrimiento, expresión y sumisión.

Cada uno de estos elementos, así como sus combinaciones, deben adaptarse al grupo-clase en el que se vaya a gamificar el aprendizaje, ya que en función del diseño concreto será más importante la presencia de unos $\mathrm{u}$ otros elementos (Deterding et al., 2011). Valga como ejemplo el caso de los juegos de rol, donde componentes como los avatares son indispensables. Hecho que ensalza la importancia de adecuar al contexto y población los elementos de juego que se van a implementar (Strmečki et al., 2015). Así por ejemplo, los socializadores se involucrarán en sistemas con dinámicas basadas en la socialización y con mecánicas que impliquen rankings de clasificación; por su parte los filántropos se motivarán ante componentes como las colecciones; o los triunfadores preferirán sistemas en los que predominen componentes como niveles o combates y dinámicas de aprendizaje de habilidades (Tondello et al., 2016).

De lo expuesto anteriormente se deriva el énfasis que se debe poner en la metodología al trabajar con herramientas como los sistemas gamificados, ya que su incorrecta planificación y ejecución puede llevar al fracaso de la propuesta (Teixes, 2014). Se destacan algunos aspectos a tener en cuenta planteados por Chavez-Yuste (2019) como la importancia de que los elementos de juego comulguen con el alumnado, la necesidad de encontrar un equilibrio entre la motivación intrínseca y extrínseca, así como entre la cooperación y la competición, promoviendo la sana competición donde se fomente el aprendizaje por medio de la utilización de recompensas insustanciales (Cantador-Guitiérrez, 2016), el diseño de mecánicas que superen el nivel del que parte el estudiante, para motivarlo a superarse pero sin desanimarlo con tareas inasequibles, e ir manteniendo dicha motivación por medio de recompensas al progreso, o lo que es lo mismo componentes que proporcionen feedback a corto plazo que le permitan llegar al objetivo final.

A modo de resumen destacar que para poner en funcionamiento un sistema gamificado es necesario en primer lugar realizar una evaluación inicial que identifique las características de nuestro alumnado (García-Casaus et al., 2020) y definir nuestros objetivos de aprendizaje. En función de dichos resultados y de forma consensuada con el alumnado definir componentes, mecánicas y dinámicas del sistema, así como una estética a utilizar acorde al contenido (Chaves-Yuste, 2019). Por último, una vez implementado el sistema es necesario mantener una evaluación continua del proceso para poder mejorarlo, reestructurarlo y actualizarlo (Rughiniş, 2013).

\section{HERRAMIENTAS TIC PARA GAMIFICAR LA DOCENCIA}

Una vez abordada la conceptualización de gamificación, sus beneficios y cómo llevarla a cabo, es necesario conocer algunas herramientas TIC que favorecen su integración en el aula. Si utilizamos un modelo de gamificación estructurado en nuestras aulas, el alumnado controlará los estímulos del contenido, será capaz de decidir qué puntos/premios desea obtener, o contra qué-quienes quiere competir, además la interactividad con los contenidos contribuye a disminuir la desmotivación y generar nuevas oportunidades de reflexión (Kapp et al., 2014).

A continuación, presentamos las principales aplicaciones TIC que pueden utilizarse para gamificar las aulas de forma lúdica, motivadora y pedagógica (educación 3.0, 2020). No se trata de un listado exhaustivo, más bien actúa como una guía ejemplo de la que partir, que puede verse incrementada posteriormente por los propios docentes, pues existen más recursos sobre gamificación en la propia red. Además de proporcionar información, estas aplicaciones son analizadas y clasificadas atendiendo a tres categorías, con la finalidad de favorecer la toma de decisiones docentes en la selección de qué dinámica emplear en clase. 
Tabla 1. Clasificación de herramientas que facilitan los procesos de gamificación.

Plataformas con juegos y recursos educativos
Cerebrity. Plataforma en la que aparece un catálogo de juegos educativos y además se ofrece la posibilidad de que los alumnos creen sus propios juegos.

icuadernos. Aplicación en la que aparecen disponibles cuadernos online con los que trabajar los contenidos de las materias instrumentales.

Elever. Promueve el hábito diario para que el aprendizaje de los alumnos se consiga con ejercicios breves pero de forma continua.

MyClassGame. Permite importar cursos de Google Classroom en los que pueden participar todos los alumnos del aula. Edmodo Gamificación. Aplicación en la que el docente plantea actividades y retos, es muy motivadora, ya que no solo premia al mejor, sino que valora otros aspectos.

Monster Kit. Es una aplicación con un formato parecido al de los juegos de mesa, orientado para que los alumnos trabajen lenguaje, matemáticas y creatividad de forma lúdica.

Arcademics. Página web en la que se recogen juegos online interactivos donde pueden jugar varios compañeros a la vez.

Plataformas de videojuegos educativos

Ta-tum. Aplicación en la que participan detectives, cuya misión es guiar a los alumnos para que se conviertan en detectives literarios, para ello, pueden acceder a una biblioteca en la que hay muchos títulos.

Knowre. Videojuego que promueve el trabajo con contenidos matemáticos, incluye retos sobre álgebra y geometría, y busca ser un complemento para la clase presencial más estática.

Minecraft, EducationEdition. Es una de las aplicaciones favoritas de docentes y alumnos, su versatilidad hace que sea una herramienta apropiada.

Classcraft. El conocido juego 'World of Warcraft' para educación en clase, muy motivador.

ChemCaper. Videojuego en el que a través de experimentos se trabaja la asignatura de química.

Plataformas Toovari. Aplicación en la que se trabajan con problemas de distintas materias, fomenta la participación y el aprendizaje basadas en $\mathrm{ABJ}$ colaborativo.

reales

Kahoot! Es una herramienta que sirve para crear retos de preguntas y respuestas.

Play Brighter. Aplicación para gamificar el aula donde el docente plantea las características del juego a seguir.

Quizizz. Permite crear preguntas tipo test que se hacen en clase en tiempo real.

Trivinet. El conocido trivial en versión digital.

Genially. Una multiherramienta con diversos contenidos pensados para gamificar el aula.

En la primera categoría se contemplan las plataformas que recopilan varios juegos y recursos educativos ampliamente utilizados por los docentes, con un elevado potencial motivador constatado por los usuarios y fáciles de utilizar. En segundo lugar, se han aglutinado los videojuegos educativos que pueden ser empleados con fines didácticos dentro del aula, a pesar de que inicialmente, la mayoría de estos recursos no fueron diseñados con esa finalidad. No obstante, el paso a una red cada vez más activa en la creación digital, que permite incluso customizar lo creado por otros, favorece el diseño de juegos simples que se ajusten a los contenidos curriculares. Al respecto, Flores (2019) realiza un estudio donde trabaja los contenidos curriculares de la asignatura de educación física mediante la aplicación del juego Super Mario Bros. Asimismo, la vinculación de los jóvenes con este tipo de recursos que forman parte de su vida cotidiana constituye una fortaleza educativa que debe ser aprovechada por los docentes, pues como señala Trejo (2019), permiten fomentar su implicación y participación activa en la dinámica del aula. En este sentido, son una herramienta con mucho potencial a nivel de motivación para los alumnos, los niveles de implicación en este sentido son amplios y el aprendizaje que se produce también. Por último, las plataformas basadas en $A B J$ reales tienen el componente lúdico y el formato atractivo que "engancha" a los alumnos para aprender cada vez más y mejor.

El listado de herramientas expuestas no desempeña un papel meramente contemplativo en la alfabetización del docente, consistente en conocer la utilidad de múltiples formas de gamificar, por el contrario, su funcionalidad radica en operativizar actuaciones. Esto es posible si los docentes son capaces de superar la visión negativa que en muchas ocasiones se genera cuando se mencionan este tipo de aplicaciones, buscando nuevas prácticas educativas para responder a las exigencias de la sociedad actual, particularmente de nuestro alumnado y lograr nuevos diseños que promuevan la consecución de aprendizajes significativos y se conviertan en herramientas 
potenciadoras del cambio educativo (Parra-González et al., 2020).

La tendencia educativa actual, nos muestra que el problema no está en la utilización de tecnología y en particular de los videojuegos, sino en el uso que se les asigna en el proceso de enseñanza-aprendizaje, tanto formal como informal. Las potencialidades de los juegos digitales para el aprendizaje son numerosas, y las investigaciones realizadas constatan mejoras importantes en la implicación de los alumnos siendo utilizado para el entrenamiento y comprensión de situaciones complejas. Además, se ha evidenciado que facilitan el aprendizaje $\mathrm{y}$, usados adecuadamente, fortalecen valores y actitudes muy necesarios para la socialización de los alumnos (Kapp, 2012).

\section{CONCLUSIONES}

Como se ha puesto de manifiesto a lo largo de este artículo, la gamificación a través del uso de las TIC se ha convertido en un recurso educativo que ha demostrado ser útil y por el que se está tomando cada vez más interés (Reyes-Cabrera \& Quiñones, 2020). La actual evolución que han experimentado los videojuegos y las aplicaciones digitales ha demostrado tener un gran potencial didáctico en las aulas, razón por la que los docentes cada vez más emplean esta metodología (Basten, 2018).

Con la utilización de las herramientas digitales como eje central de la gamificación, se consigue que los alumnos no vean el proceso educativo como una obligación, puesto que, gracias al componente lúdico y al protagonismo de medios que el alumnado domina, la experiencia de aprendizaje se convierte en algo divertido y motivador. Concretamente, según Torres-Toukoumidis et al. (2018), esta modalidad metodológica se ajusta a las siguientes necesidades educativas: concebir al alumno como protagonista de la acción educativa; promover un aprendizaje activo que contribuye a aumentar los niveles de motivación; aprender por inmersión al introducirse el alumno en el contexto de juego, llegando incluso a asumir el rol de otra persona; aprender colaborativamente integrando y normalizando la utilización de las TIC en todos los contextos educativos; favorecer el desarrollo de la imaginación y la creatividad; finalmente, permitir evaluar los contenidos teórico-prácticos del alumno, por lo que constituyen una alternativa didáctica que también favorece una evaluación integral de los aprendizajes. Otros estudios, afirman que las dimensiones teóricas propuestas desde la gamificación se adaptan a la valoración que hacen los profesionales de la enseñanza sobre las necesidades de la enseñanza E-learning, entre los que destacan una serie de indicadores: contrastar las habilidades que según la pedagogía tradicional deben trabajarse con las propuestas por la pedagogía moderna, comprobar el rol que desempeña el docente en la metodología didáctica y estimar la presencia de otros actores en el proceso de enseñanza, valorar la interacción del alumno y el docente con los recursos empleados, extraer los componentes lúdicos de la estrategia pedagógica y finalmente, facilitar la autonomía del alumnado en la toma de decisiones (Torres-Toukoumidis et al., 2017).

Son muchos los estudios que destacan los beneficios de esta metodología, así la Universidad de Montreal realizó un análisis de la literatura y concluyó que varios aspectos ponen de manifiesto su efectividad, desde mejoras cognitivas -mejoras en retención del conocimiento y habilidades de pensamiento-, comportamentales -aumento de la asistencia y participación en el aula-, emocionales -aumento de la motivación, disminución de ansiedad ante el error-, hasta sociales -aumento de la colaboración, disminución de las diferencias entre alumnos con distintas capacidades- (Rabah et al., 2018).

La consecución de óptimos resultados con la aplicación de esta metodología responde a un esfuerzo por parte del docente, de abandonar la didáctica tradicional y pasar a acompañar al alumno en un proceso de creación de conocimiento, que deja de ser meramente memorístico y pasa a basarse en la solución de problemas (García-Casaus et al., 2020). Para ello es fundamental partir de las necesidades del alumnado y adaptar adecuadamente las mécanicas, dinámicas y elementos de juego al proceso educativo concreto, en palabras de Chavez-Yuste "debe haber un equilibrio entre actividad lúdica, destrezas personales, conocimientos previos, adquisición de nuevos contenidos y desafíos" (2019, p.429).

Independientemente de la procedencia del juego que se utilice en el aula, la inclusión de dinámicas gamificadas en las aulas para facilitar el proceso de aprendizaje del alumnado debe ser una premisa en todos los centros educativos, tanto públicos como privados, puesto que los juegos han demostrado ser una opción didáctica cercana, efectiva y motivadora para el aumento del interés y la motivación (Torres-Toukoumidis et al., 2021).

Como conclusión se destaca que cada vez es más evidente la necesidad de vincular las prácticas educativas a la sociedad digital: no se puede seguir aprendiendo con las mismas reglas del pasado y negando o volviendo la espalda a la sociedad cambiante y trasformadora. Los docentes tienen el compromiso moral de formarse y contar con las estrategias y recursos más adecuados a las motivaciones e intereses del alumnado. El cambio del rol del docente y del alumnado en una sociedad que demanda el acceso a la tecnología es un hecho consumado. La autonomía del 
alumno y el protagonismo de su propio aprendizaje guiado por un profesor, que es orientador, consultor y tutor, más que transmisor de conocimientos teóricos, es una realidad asentada por estudiosos e investigadores en educación. Este cambio debe ser acompañado de procesos y entornos formativos gamificados, en los que el aprendizaje de los contenidos disciplinares y transversales (valores, emociones, ciudadanía, ...) se vuelvan atractivos al alumnado. La combinación de la gamificación y las TIC permite al estudiante una experiencia de aprendizaje significativo, basado en la autonomía de la construcción del conocimiento conectado con la realidad a través de la relación con los contenidos y materiales de forma personal en un entorno digitalizado. Todo ello con el objetivo de avanzar en el cambio del proceso de enseñanza-aprendizaje de estudiantes que son nativos digitales.

\section{REFERENCIAS}

Acevedo, J.A., Caicedo, E., \& Ferney, J. (2019). Beneficios del Ambiente Robótico Lúdico SpinBOT en el Desarrollo de Habilidades Cognitivas. Revista Iberoamericana de Automática e Informática, 16(2), 171-177. https://doi.org/10.4995/riai.2017.8763

Aparici, R., \& Osuna-Acedo, S. (2010). Educomunicación y cultura digital. En R. Aparici (ed.), Educomunicación: más allá del 2.0 (pp. 307-318). Edisa Editorial, S.A.

Apostol, S., Zaharescu, L., \& Alexe, I. (2013). Gamification of learning and educational games. eLearning \& Software for Education, 2, 67-72.

Basten, D. (2018). Gamification. The IEEE Computer Society, 34(5), 76-81.

Bonilla del Río, M., Garcia-Ruiz, R., \& Diego-Mantecón, J. M. (2018). Gamificación en la escuela 2.0: una alianza educativa entre juego y aprendizaje. En García-Ruiz, R., Bonilla-del-Río, M. \& Diego-Mantecón, J.M. (Coord.) Gamificación en Iberoamérica. Experiencias desde la comunicación y la educación (pp-71-95). Editorial Abya-Yala.

Bunchball, I. (2010). Gamification 101: An introduction to the use of game dynamics to influence behavior. White paper, 9. 1-18. http://jndglobal.com/wp-content/uploads/2011/05/gamification1011.pdf

Cantador-Gutiérrez, I. (2016). La competición como mecánica de gamificación en el aula: una experiencia aplicando aprendizaje basado en problemas y aprendizaje cooperativo. En: Contreras-Espinosa, R, \&Eguia-Gómez, J. L. (Ed.). Gamificaciónen aulas universitarias. Bellaterra: Institut de la Comunicació: UniversitatAutònoma de Barcelona, 68-97.
Contreras, R. S., \& Eguia, J. L. (2016). Gamificación en aulas universitarias. Institut de la Comunicació.

Cózar, R. y De Moya, M.V. (coords.) (2013). Las TIC en el aula desde un enfoque multidisciplinar. Octaedro.

Chaves-Yuste, B. (2019). Revisión de experiencias de gamificación en la enseñanza de lenguas extranjeras. ReiDoCrea, 8, 422-430. https://www.ugr. es/ reidocrea/8-33.pdf

Deterding, S., Dixon, D., Khaled, R. y Nacke, L. (2011, septiembre). From Game Design Elements to Gamefulness: Defining Gamification. En Proceedings of the 15th International Academic MindTrek Conference: Envisioning Future Media Environments. $10.1145 / 2181037.2181040$

Díaz-Cruzado, J., \& Troyano, Y. (2013). El potencial de la gamificación aplicado al ámbito educativo. III Jornadas de Innovación Docente. Innovación Educativa: respuesta en tiempos de incertidumbre. https://idus. us.es/handle/11441/59067

Dicheva, D., Dichev, C., Agre, G., \& Angelova, G. (2015). Gamification in education: a systematic mapping study. Educational Technology \& Society, 18(3), 75-88. https://www.jstor.org/stable/jeductechsoci.18.3.75

Educación 3.0. (2020, mayo 20). 27 herramientas de gamificación para clase que engacharán a tus alumnos. Recuperado el 12 dicembre 2020 de https://www. educaciontrespuntocero.com/recursos/herramientasgamificacion-educacion/

Elías, A., \& González, A.M. (2019). Formación Docente: aportaciones para un estado del arte desde una perspectiva participativa. Investigación Cualitativa, 4(1), 75-90. https://ojs.revistainvestigacioncualitativa.com/ index.php/ric/article/view/115

Escribano, F. (2013). Gamificación versus ludictadura. Obra digital, 5, 58-72. https://doi.org/10.25029/ od.2013.22.5

Fernández-Arias, P., Ordónez-Olmedo, E., VergaraRodríguez, D., \& Gómez-Vallecillo, A.I. (2020). La gamificación como técnica de adquisición de competencias sociales. Prisma Social, 31, 388-409. https:// revistaprismasocial.es/article/view/3698

Flores, G. (2019). ¿Jugamos al Súper Mario Bros? Descripción de una experiencia gamificada en la formación del profesorado de Educación Física. Retos, 36, 529534. https://doi.org/10.47197/retos.v36i36.67816

Furdu, I., Tomozei, C., y Kose, U. (2017). Pros and cons gamification and gaming in classroom. Broad Research in Artificial Intelligence and Neuroscience, 8, 56-62.

García-Ávila, S. (2017). Alfabetización digital. Razón y Palabra, 21(3_98), 66-81. https://www.revistarazonypalabra.org/index.php/ryp/article/view/1043 
García-Casaus, F., Cara-Muñoz, J.F., Martínez-Sánchez, J.A., \& Cara-Muñoz, M.M. (2020). La gamificación en el proceso de enseñanza-aprendizaje: una aproximación teórica. Logía: EducaciónFísica y Deporte 2020, 1(1), 16-24. https://logiaefd.com/revista-no1-volumen-1-logia-educacion-fisica-y-deporte

Garone P., \& Nesteriuk S. (2019) Gamification and Learning: A Comparative Study of Design Frameworks. In: Duffy V. (eds) Digital Human Modeling and Applications in Health, Safety, Ergonomics and Risk Management. Healthcare Applications. HCII 2019. Lecture Notes in Computer Science, vol 11582. Springer, Cham. https://doi.org/10.1007/978-3-030-22219-2_35

Gil, J., \& Prieto, E. (2019). Juego y gamificación: Innovación educativa en una sociedad en continuo cambio. Revista Ensayos Pedagógicos, 14(1), 91-121. https://doi.org/10.15359/rep.14-1.5

Gómez-Vargas, M., Galeano, C., \& Jaramillo, D.A. (2015). El estado del arte: una metodología de investigación. Revista Colombiana de Ciencias Sociales, 6(2), 423442. https://doi.org/10.21501/22161201.1469

Guillén-Rascón, G., Ascencio-Baca, G., \& Tarango, J. (2016). Alfabetización digital: Una perspectiva sociológica. E-Ciencias de la Información, 6(2), 96-116. https://doi.org/10.15517/eci.v6i2.23938

Guzmán, M., \& García-Garduño, J.M. (2016). Determinantes que afectan la enseñanza de la metodología de las ciencias sociales: un estado del arte. Perfiles educativos, 38(153), 51-64. https://doi.org/10.22201/ iisue.24486167e.2016.153.57635

Hamari, J., Shernoff, D. J., Rowe, E., Coller, B., AsbellClarke, J., \& Edwards, T. (2016). Challenging games help students learn: An empirical study on engagement, flow and immersion in game-based learning. Computers in Human Behavior, 54,170-179. https:// doi.org/10.1016/j.chb.2015.07.045

Hammer, J., \& Lee, J. J. (2011). Gamification in Education: What, How, Why Bother? Academic Exchange Quarterly, 15(2), 1-5.

Hunicke, R., Leblanc, M. \& Zubek, R. (2004). MDA: A Formal Approach to Game Design and Game Research. AAAI Workshop on Challenges in game AI - Technical Report 1. http://ksuweb.kennesaw. edu/ jprest20/cgdd2002/MDA.pdf

Iglesias-Forneiro, M. L. (2008). Observación y evaluación del ambiente de aprendizaje en educación infantil: dimensiones y variables a considerar. Revista Iberoamericana de educación, 47(3), 49-70. https://core. ac.uk/download/pdf/41562864.pdf

Kapp, K.M., Blair, L., \& Mesch, R. (2014). The Gamification of Learning and instruction Fieldbook - Ideas into Practice. Wiley.
Kapp, K. (2012). The Gamification of Learning and Instruction. Nueva York: ASTD.

Kiryakova, G., Angelova, N., \& Yordanova, L. (2014). Gamification in education [Presentación en congreso]. 9th International Balkan Education and Science Conference. Edirne, Turquía.

Lamoneda, J., González-Víllora, S., \& Fernández-Río, J. (2020). Hibridando el Aprendizaje Cooperativo, la Educación Aventura y la Gamificación a través de la carrera de orientación (Hybridizing Cooperative Learning, Adventure Education, and Gamification through orienteering races). Retos, 38, 754-760. https://doi.org/10.47197/retos.v38i38.77276

Levis, D. (2006). Alfabetos y saberes: la alfabetización digital. Comunicar: Revista científica iberoamericana de comunicación y educación, 26, 78-82. https://doi. org/10.3916/C26-2006-12

Llorens-Largo, F., Gallego-Duran, F. J., Villagra-Arnedo, C. J., Compan-Rosique, P., Satorre- Cuerda, R., \& Molina-Carmona, R. (2016). Gamification of the Learning Process: Lessons Learned. IEEE RevistaIberoamericana de Tecnologias Del Aprendizaje, 11(4), 227-234. https://doi.org/10.1109/RITA.2016.2619138

Londoño, O. L., Maldonado, L., \& Calderón, L. (2014). Guías para construir estados del arte. International Corporation of Networks of Knowledge https://iconk. org/docs/guiaea.pdf

Marczewski, A. (2015). User Types. En A. Marczewski Even Ninja Monkeys Like to Play: Gamification, Game Thinking and Motivational Design ( ${ }^{\mathrm{a}}$ ed., pp. 65-80). CreateSpaceIndependent Publishing Platform.

Miller, C. (2013). The gamification of education. Developments in Business Simulation and Experiential Learning, 40, 196-200. https://absel-ojs-ttu.tdl.org/absel/ index.php/absel/article/view/40

Molina, P., Valenciano, J, \& Valencia-Peri, A. (2015). Los blogs como entornos virtuales de enseñanza y aprendizaje en Educación Superior. Revista Complutense de Educación, 26, 15-31. http://dx.doi. org/10.5209/rev_RCED.2015.v26.43791

Molina-Montoya, N. P (2005). ¿Qué es el estado del arte?. Ciencia y Tecnología para la salud Visual y Ocular, 5, 73-75. https://doi.org/10.19052/sv.1666

Molina-Ríos, J. R., Zea, M.P., Contento, M.J., \& García-Zerda, F.G. (2017). Estado del arte: Metodologías de desarrollo en aplicaciones web. 3c Tecnología, 6(3), 54-71. http://dx.doi.org/10.17993/3ctecno.2016.v6n3e23.54-71

Mominó, J.M. \& Sigalés, C. (Coords.). (2016). El impacto de las TIC en la Educación. Más allá de las promesas. UOC Ediciones.

Naismith, L., Lonsdale, P., Vavoula, G., \& Sharples, M. (2004). Literature Review in Mobile Technologies and 
Learning: Report 11. https://hal.archives-ouvertes.fr/ hal-00190143/document

Parra-González, M.E., Segura-Robles, A., \& RomeroGarcía, C. (2020). Análisis del pensamiento creativo y niveles de activación del alumno tras una experiencia de gamificación. Educar, 56(2). https://doi. org/10.5565/rev/educar.1104

Pertegal, M.L. \& Lorenzo, G. (2019). Gamificación en el aula a través de las TIC. International Journal of Developmental and Educational Psychology INFAD Revista de Psicología,1 (Monográfico 2), 553562. https://doi.org/10.17060/ijodaep.2019.n1.v3.1535

Prensky, M. (2009). H. sapiens digital: From immigrants and digital natives to digital wisdom. Innovate: Journal of Online Education, 5 (3). https://nsuworks.nova. edu/innovate/vol5/iss $3 / 1$

Prieto-Andreu, J.M. (2020). Una revisión sistemática sobre gamificación, motivación y aprendizaje en universitarios. Teoría de la Educación. Revista Interuniversitaria, 32(1), 73-99. https://doi.org/10.14201/ teri.20625

Qian, M. \& Clark, K.R. (2016). Game-based Learning and 21st century skills: A review of recent research. Computers in human behavior, 63, 50-58. https://doi.org/10.1016/j.chb.2016.05.023

Quintanal, F. (2016). Aplicación de herramientas de gamificación en física y química de secundaria. Opción: Revista de Ciencias Humanas y Sociales, 12, 327-348. http://www.redalyc.org/articulo.oa?id=31048903016

Rabah, J., Cassidy, R., \& Beauchemin, R. (2018). Gamification in education: Real benefits or edutainment? European Conference on e-learning. https://doi. dx.org/10.13140/RG.2.2.28673.56162

Rackwitz, R. (2012, Octubre 8). Why Gamification is more than just a trend. Engaginglab. https://engaginglab.wordpress.com/2012/10/08/why-gamificationis-more-than-just-a-trend/

Real Academia Española. (2019). Ludificación. En Diccionario de la Lengua Española. Recuperado el 20 de diciembre de 2020 de: https://www.rae.es/observatorio-de-palabras/ludificacion

Reyes, C.E. (2019). Estrategia metodológica para elaborar un estado del arte como un producto de investigación científica. Praxis Educativa, 23(3), 1-14. https://dx.doi.org/10.19137/praxiseducativa-2019-230307

Reyes-Cabrera, W.R., \& Quiñonez, S.H. (2020). Gamificación en la educación a distancia: experiencias en un modelo educativo universitario/Gamification in distanceeducation: experiences in a universityeducationalmodel. Apertura:Revista de Innovación Educativa, 12(2). http://dx.doi.org/10.32870/Ap.v12n2.1849
Rivero, M.P. (2017). Procesos de gamificación en el aula de Ciencias Sociales. Iber: Didáctica de las ciencias sociales, geografía e historia, 86, 4-6. https://www. grao.com/es/producto/procesos-de-gamificacion-enel-aula-de-ciencias-sociales

Rodríguez-García, F., \& Santiago-Campión, R. (2015). Gamificación: Cómo motivar a tu alumnado y mejorar el clima en el aula. Grupo Océano.

Rughiniş, R. (2013). Flexible gamification in a social learning situation. Insights from a collaborative review exercise. En N. Rummel, M. Kapur, M. Nathan, \& S. Puntambekar (Eds.), 10th International Conference on Computer Supported Collaborative Learning CSCL 2013. Conference Proceedings Volume II (pp. 137-140). International Society of the Learning Sciences (ISLS).

Sánchez, F. J. (2015). Gamificación. Education in the Knowledge Society, 16(2), 13-15. https://doi. org/10.14201/eks20151621315

Strmečki, D., Bernik, A., \& Radošević, D. (2015). Gamification in e-learning: Introducing gamified design elements into e-learning systems. Journal of Computer Science, 11(12), 1108-1109. https://doi.org/10.3844/ jcssp.2015.1108.1117

Teixes, F. (2015). Gamificación: Motivarjugando. Barcelona: Editorial UOC.

Teixes, F. (2014). Gamificación: Fundamentos y aplicaciones. Barcelona: Editorial UOC.

Tondello, G.F., Mora, A., Marczewski, A., \& Nacke, L.E. (2019). Empirical validation of the Gamification User Types Hexad scale in English and Spanish. International Journal of Human-Computer Studies, 127, 95-111. https://doi.org/10.1016/j.ijhcs.2018.10.002.

Tondello, G.F., Wehbe, R., Diamond, L., Busch, M., Marczewski, A., \& Nacke, L.E. (2016). The Gamification User Types Hexad Scale. En Proceedings of the 2016 Annual Symposium on Computer-Human Interaction in Play(CHI PLAY '16). Association for Computing Machinery, New York, NY, USA, 229-243. https://doi. org/10.1145/2967934.2968082

Torres-Toukoumidis, A., Carrera, P., Balcazar, I., \& Balcazar, G. (2021). Descriptive Study of Motivation in Gamification Experiences from Higher Education: Systematic Review of Scientific Literature. Universal Journal of Educational Research, 9(4), 727-733. https://doi.org/10.13189/ujer.2021.090403

Torres-Toukoumidis, A., Romero-Rodriguez, L.M, \& Perez-Rodriguez, A. (2018). Ludificación y sus posibilidades en el entorno de blended learning: revisión documental. Revista Iberoamericana de Educación a Distancia 21(1), 95-111. https://doi.org/10.5944/ ried.21.1.18792 
Torres-Toukoumidis, A., Romero-Rodríguez, L., PérezRodríguez, M.A., \& Björk, S. (2017). Modelo Teórico Integrado de Gamificación en Ambientes E-Learning (E-MIGA). Revista Complutense De Educación, 29(1), 129-145. https://doi.org/10.5209/RCED.52117

Torres Toukoumidis, A., Romero-Rodríguez, L.M., Pérez-Rodríguez, M. A., \& Björk, S. (2016). Desarrollo de habilidades de lectura a través de los videojuegos: Estado del arte. Ocnos: Revista de estudios sobre lectura, 15(2), 37-49. http://doi.org/10.18239/ ocnos_2016.15.2.1124

Trejo González, H. (2019). Technological resources for the integration of gamification in the classroom. Tecnología, Ciencia y Educación, 13, 75-117. https://tecnologia-ciencia-educacion.com/index.php/TCE/article/ view/285

UNESCO (2011). Competency Framework for Teachers. http://unesdoc.unesco.org/images/0021/002134/ 213475e.pdf

Villena, R., Cózar, R. \& González-Calero, J.A. (2108). Gamificación en grupos interactivos para enseñar y aprender historia. Una experiencia en educación primaria. En José Monteagudo-Fernández, Ainoa Escribano-Miralles y Cosme J. Gómez-Carrasco (Eds.). Educación histórica y competencias transversales: narrativas, TIC y competencia lingüistica. Editum.

Werbach, K., \& Hunter, D. (2012). For the Win: How Game Thinking can Revolutionize your Business. University of Pennsylvania PressWharton Digital Press. 